\title{
EVALUATION OF FOSFOMYCIN AND LASER IN THE TREATMENT OF METHICILLIN-RESISTANT STAPHYLOCOCCUS AUREUS (MRSA) OSTEOMYELITIS IN RABBITS
}

\author{
Walaa Samir Abdel-Fatah* and Raghda Hager**
}

\begin{abstract}
Aim: to evaluate the effectiveness of fosfomycin and Diode laser treatment in experimental osteomyelitis induced by MRSA in rabbits.

Methods: Osteomylitis Infection was induced in the tibiae of thirty rabbits by the injection of a bacterial inoculum $\left(7 \times 10^{6} \mathrm{CFU} / \mathrm{ml}\right)$ of a clinical MRSA isolate two weeks after infection, each animal was assigned to a treatment group: Group (1) include 10 animals were left untreated Group (2); Fosfomycin monotherapy at $75 \mathrm{mg} / \mathrm{kg}$ of body weight once daily (n_10), Group 3: (n = 10) rabbits were treated for 2 weeks with Diode laser $940 \mathrm{~nm}$.After a 2 -week treatment period, the animals were euthanized, and the infected tibiae were processed for quantitative bacterial cultures and bone density measurement .
\end{abstract}

RESULTS: bone culture from bone was positive for MRSA in all groups with no significant difference ( $p$ value $<0.302$ ). Based on bone density measurement; bone density was higher level at laser group followed by antibiotic FOF group then control group but there is no with significant difference $\mathrm{p}$ value $<0.15$. Correlation between bacterial count and bone density yield significant difference in control group with $\mathrm{p}$ value $<0.03$.

Conclusion: The difference between laser group and fosfomycin group not significantly different between the two methods for modeling rabbit. However, histopathological method is recommended because it has certain increase confirmation which line of treatment was viald.

KEYWORDS: Rabbit, MRSA, Osteomylitis, Bone density, Diode laser, Digora and Fosfomycin.

\section{INTRODUCTION}

Osteomyelitis is a bone infection that can initiate due to a nearby trauma or infection. The microorganisms chargeable for the infection are typically pyogenic bacteria.The most frequent infectious agents are inorder of occurrence Staphylococcus aureus (S. aureus), Streptococcus (groups A and B), Haemophilus influenzae, and Enterobacter species ${ }^{(14)}$.

* Lecturer of Oral Maxillofacial Radiology; Faculty of Dentistry , Bani-seuif University.

** Lecturer of Microbiology and Immunology ;Faculty of medicine, Misr university For science and technology. 
Staphylococcus aureus ( $S$. aureus) is the most commen pathogen causing osteomyelitis ${ }^{(\mathbf{1})}$. The growing number of methicillin-resistant Staphylococcus aureus (MRSA) isolates reduces the antimicrobial treatment alternatives ${ }^{(2)}$. Vancomycin has been used for treatment of MRSA since 1954 and it is the drug of choice within the treatment serious MRSA infections worldwide ${ }^{(3)}$. The emergence of vancomycin resistance represents a prime problem in the remedy of deep-seated infections, such asosteomyelitis ${ }^{(4)}$ so there are an pressing need for alternative antibiotics.

Fosfomycin (FOF) a broad-spectrum antibacterial agent owning a completely unique chemical structure, particular pharmacologic functions and a loss of toxicity, is a promising drug for scientific use. it's miles lively in opposition to diverse Gram positive and Gram negative bacteria, which includes difficult-to-deal with pathogens inclusive of MRSA, penicillin-resistant pneumococci, and prolonged spectrum- lactamase-producing Enterobacteriaceae ${ }^{(5,6)}$. FOF is bactericidal and inhibits bacterial cell wall biogenesis ${ }^{(7)}$.

FOF has particular pharmacological traits and penetrates nicely into osseous tissue ${ }^{(8)}$. It has proved to be clinically useful for the treatment of acute and persistent osteomyelitis ${ }^{(9)}$. FOF has been shown quite powerful within the treatment of experimental

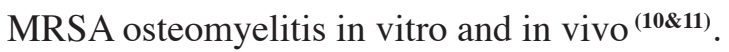

The rapid development of laser technology and wide range of characteristics new lasers are now available and being used in various fields of dentistry ${ }^{(13)}$. laser energy in differing wavelengths and irradiation regimes has a potential bactericidal effect on Staphylococcus aureus. laser phototherapy with the appropriate irradiation parameters appears to be a promising adjunct and/or alternative technique to pharmacological agents in the treatment of osteomyelitis ${ }^{(15)}$.

\section{MATERIALS AND METHODS}

Animals: The study was done in compliance with national and international standards for the protection of animals used for scientific purposes, and the project was approved by the ethics committee of the faculty of veterinary, Cairo University, Egypt. The experiment was conducted in the faculty of veterinary, Cairo University, Egypt. The animals that were used were New Zealand White rabbits, male and female. The rabbit mean age at the beginning of the experiment was 7 months and average individual weight was 1500 g. Rabbits were maintained in a temperaturecontrolled accommodation (16-21C degree). The food and water were provided ad libitum throughout the experiment period. Accommodation was done in specific cages, 2-3 rabbits in cage.

Bacterial strain: The clinical MRSA strain used in the study was obtained from patient have bacterima undergoing treatment at Egypt Children hospital for health insurance.

In vitro susceptibility testing of Fosfomycin: The minimum inhibitory concentration (MIC) of fosfomycin for MRSA strain was determined by agar dilution supplemented with Glucose-6-phosphate $(25 \mathrm{mg} / \mathrm{L})$, according to the recommendations of the Clinical and Laboratory Standards Institute (12) and result were interpreted according to European committee on antimicrobial susceptibility testing criteria (17) (susceptible, $\leq 32 \mathrm{mg} / \mathrm{L}$; resistant, $\geq 64 \mathrm{mg} / \mathrm{L}$ ).

Induction of osteomyelitis infection: Each Rabbit was anesthetized with ketamine and xylazine, and the left hind leg was shaved and disinfected with polyvinyl pyrrolidine-iodine. An 18-gauge needle was inserted percutaneously through the lateral aspect of the left tibial metaphysis into the intramedullary cavity then $0.1 \mathrm{ml}$ of MRSA (7 x 106 $\mathrm{CFU} / \mathrm{ml}$ ).

Treatment groups: After 2 weeks as an incubation period, osteomyelitis of the tibia were confirmed radiographically. The animals groups In this study we used 30 rabbits divided into THREE groups as follows: 
Group 1: (control group, $\mathrm{n}=10$ ) was infected but left untreated for the duration of the study.

Group 2: (antibiotic group, $\mathrm{n}=10$ ) rabbits were treated for 2 weeks with fosfomycin .

Group 3: $(\mathrm{n}=10)$ rabbits were treated for 2 weeks with Diode laser $940 \mathrm{~nm}$.

Antibiotic preparation : Fosfomycin ( FOF) powder was dissolved in sterile water and administered intraperitoneally at a dose of $75 \mathrm{mg} / \mathrm{kg}$ once daily.(Fig. 1)

Laser irradiation : Deep tissue hand piece of Biolase diode laser $940 \mathrm{~nm} \pm 10 \mathrm{~nm}$ used to administrate 4 watt 480 joul for 120 second to

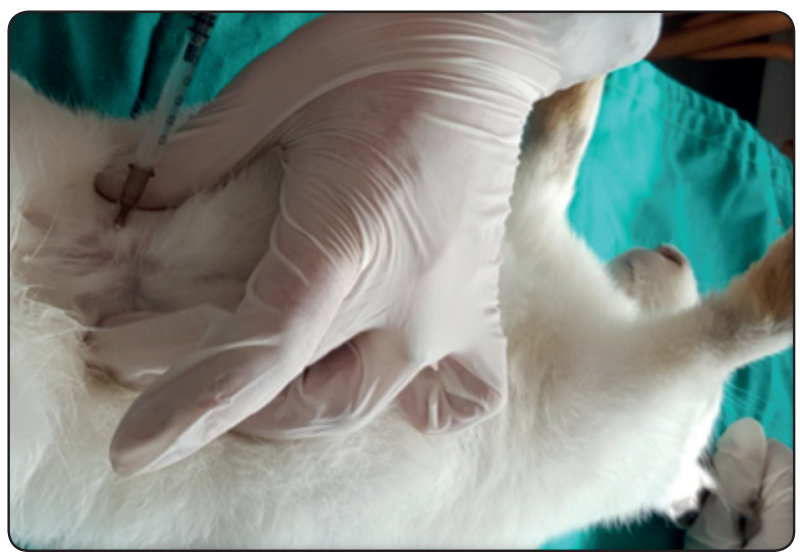

Fig. (1) Unconcious Rabbit during induction Fosfomycin antibiotic .

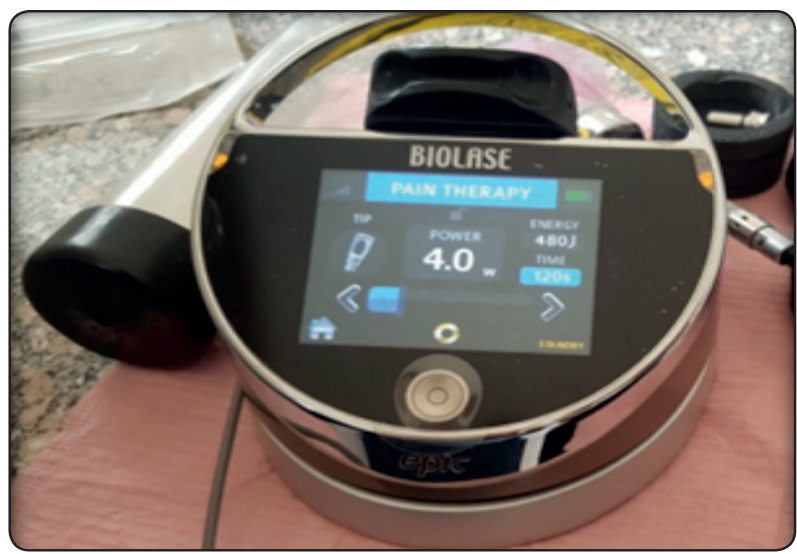

Fig. (2b) Biolase diode laser with adjusted parameters . induce biomodulation effect for two weeks 3 time in each week at site of injection infection. (Fig. 2a,2b and Fig. 3).

Bone cultures and bacterial counting: Rabbits were sacrificed 24 hours after the completion of antimicrobial therapy; both tibias were removed aseptically, dissected free of all soft tissue, and processed for bacterial cultures. The proximal and distal ends of the tibia were swabbed and streaked onto blood agar plates to check for any bacterial growth then placed into tubes containing $5 \mathrm{~mL}$ of sterile trypticase soy broth. Plates and tubes were incubated at $37^{\circ} \mathrm{C}$ for $24 \mathrm{~h}$ and growth and/or turbidity recorded.

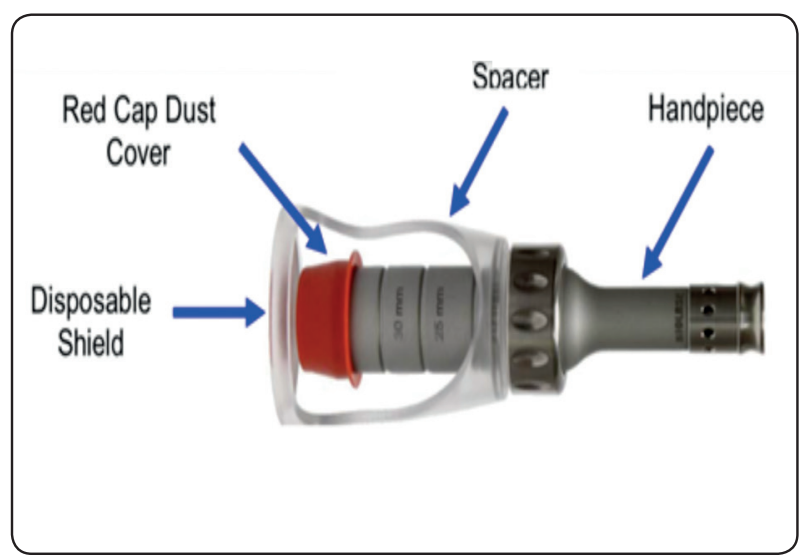

Fig. (2a) Deep tissue hand piece of Biolase diode laser

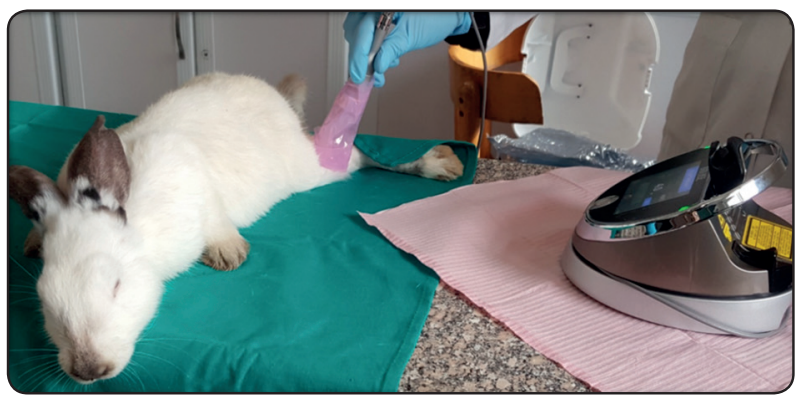

Fig. (3) Laser application to rabbit tibia . 


\section{Radiographic evaluation: Radiographic} monitoring was done by examination of both tibia of the rabbits immediately after sacrifice, using a digital image capture device photo-stimulable phosphor plates Digital Radiograph. Digitized images were manipulated using the special software of the Digora system (Orion Corporation, Soredex, Medical system, Helsinki, Finland) and bone density measured. Data Collected for all group and statically analysized. Measurement was done at different time intervals by three doctors, radiology specialists, independently of each other. (Fig4.)

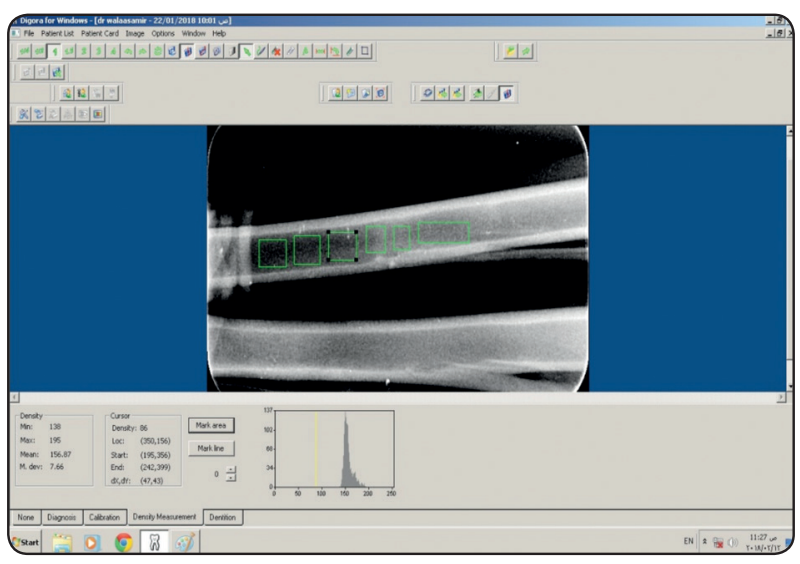

Fig. (4) Radiographic bone density measurement

\section{Statistical analysis of experimental data:}

The mean and standard deviation were calculated for all and raw data. Fisher's exact test was used to assess the significance of bacteria clearance among the treatment groups. Differences between the groups were deemed statistically significant if $\mathrm{P} \leq 0.05$.

\section{RESULTS AND DISCUSSIONS}

MICs of FOF: The MIC of the used MRSA strain was $0.5 . \mu \mathrm{g} / \mathrm{ml}$.

Treatment was began on day 14, and were not included in the data analysis. In this study, a fosfomycin monotherapy of $75 \mathrm{mg} / \mathrm{kg}$ of body weight once daily yielded no difference compared to control group and laser group .This However Schintler et al. demonstrated that the currently approved FOF dosage of $100 \mathrm{mg} / \mathrm{kg}$ body weight 2 or 3 times a day results in bone tissue concentrations that work against relevant pathogens, including MRSA $^{(8)}$. This extremely good penetration of FOF into osseous tissue will become specifically vital on the subject of reviews linking the development of bacterial resistance to subinhibitory concentrations of antibiotic on the target site ${ }^{(18,19 \& 20)}$.

FOF monotherapy was found to be effective in the treatment of experimental MRSA osteomyelitis in rats in a study done by Poeppl et al ${ }^{(10)}$.Also fosfomycin monotherapy was effective in the treatment of implant-associated and non-implantassociated osteomyelitis when fosfomycin was used in a dosage of $75 \mathrm{mg} / \mathrm{kg}^{(21)}$. fosfomycin monotherapy of $40 \mathrm{mg} / \mathrm{kg}$ of body weight once daily was superior to daptomycin or no treatment in a study done by Lingscheid et al. .22) $^{\text {(22) }}$

FOF has been used in combination and as monotherapy in a wide range of clinical settings for several decades ${ }^{(9)}$ FOF is usually combined with another antibiotic agent to prevent the development of FOF resistance ${ }^{(23)}$. The combination of daptomycin and fosfomycin has been evaluated in different experimental settings. An in vitro study demonstrated enhanced antibacterial activity of linezolid, minocycline, vancomycin, and teicoplanin against isolates of MRSA after the addition of fosfomycin (22).

In conclusion FOF was found to have potent antimicrobial activity against multiresistant Grampositive pathogens, including those with reduced susceptibility to glycopeptides, and this finding justifies the evaluation of FOF monotherapy in the treatment of osteomyelitis in humans.

\section{Bone cultures and bacterial counting:}

Bacterial cultures from bones were positive for MRSA in 8 out of $10(80 \%)$ animals in the infected untreated group, in 9 out of $10(90 \%)$ animals in the fosfomycin-treated group and 9 out of 10 (90\%) 
animals in the laser treated group, The median bacterial count in the studied group listed on table 1.

No significant difference was found in the bone cultures between the treatment groups and the infected untreated group P value 0.302. (Fig .5)

\section{Radiographic evaluation :}

Among various clinical situation bone density and bone healing may directly affect bone formation. ${ }^{(16)}$. In agreement to these reports, radiographic bone density was also assessed in the current work after laser application

TABLE (1): Bacterial counts in the studied groups
As laser phototherapy with the appropriate irradiation parameters appears to be a promising adjunct and/or alternative technique to pharmacological agents in the treatment of osteomyelitis ${ }^{(15)}$. comparing bone density between control non treated group, antibiotic group and laser group we found as in Fig. (6) that highest bone density was laser group follow by antibiotic and last was control group but statically p-value was 0.157 with non- significant difference. So, The results of present study indicate that laser phototherapy has non- significant difference effect on the healing of infected bone with MRSA(Table 2 and Figure 6).

\begin{tabular}{|c|c|c|c|c|c|c|c|c|}
\hline \multirow{2}{*}{ Groups (Infected) } & \multicolumn{6}{|c|}{ Bacterial count $\mathrm{CFU} / \mathrm{ml}$} & \multicolumn{2}{|c|}{ ANOVA } \\
\hline & \multicolumn{3}{|c|}{ Range } & Mean & \pm & SD & $\mathbf{F}$ & P-value \\
\hline Control group & 0 & - & 5 & 2.167 & \pm & 1.941 & \multirow{3}{*}{1.298} & \multirow{3}{*}{0.302} \\
\hline Antibiotics group & 0 & - & 9 & 4.667 & \pm & 3.386 & & \\
\hline Laser group & 0 & - & 7 & 4.000 & \pm & 2.828 & & \\
\hline
\end{tabular}

TABLE (2): Bone density in the studied groups:

\begin{tabular}{|c|c|c|c|c|c|c|c|c|}
\hline \multirow{2}{*}{ Groups (Infected) } & \multicolumn{6}{|c|}{ Bone density } & \multicolumn{2}{|c|}{ ANOVA } \\
\hline & \multicolumn{3}{|c|}{ Range } & Mean & \pm & SD & $\mathbf{F}$ & P-value \\
\hline Control group & 58.8 & - & 169.8 & 128.727 & \pm & 40.883 & \multirow{3}{*}{2.097} & \multirow{3}{*}{0.157} \\
\hline Antibiotics group & 114.6 & 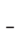 & 171.36 & 147.818 & \pm & 26.132 & & \\
\hline Laser group & 124.02 & - & 193.9 & 165.902 & \pm & 24.739 & & \\
\hline
\end{tabular}

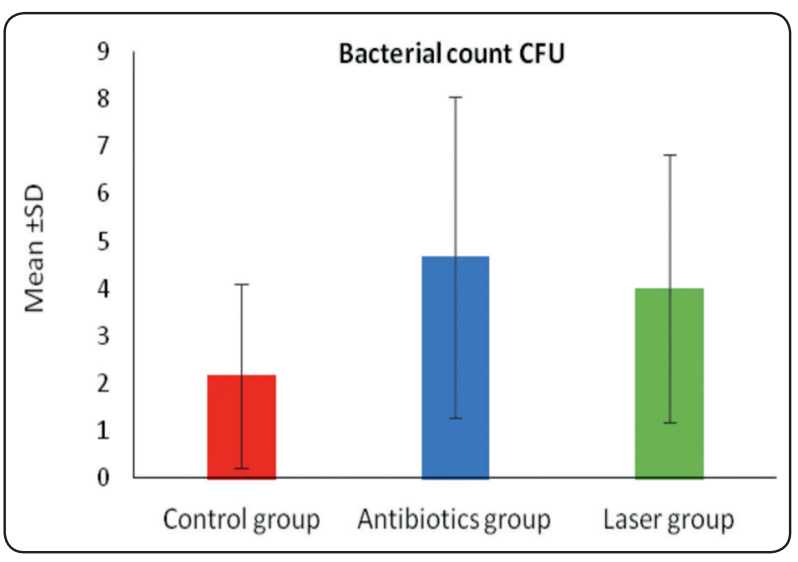

Fig. (5) Graphical representation difference in bacterial count between three group

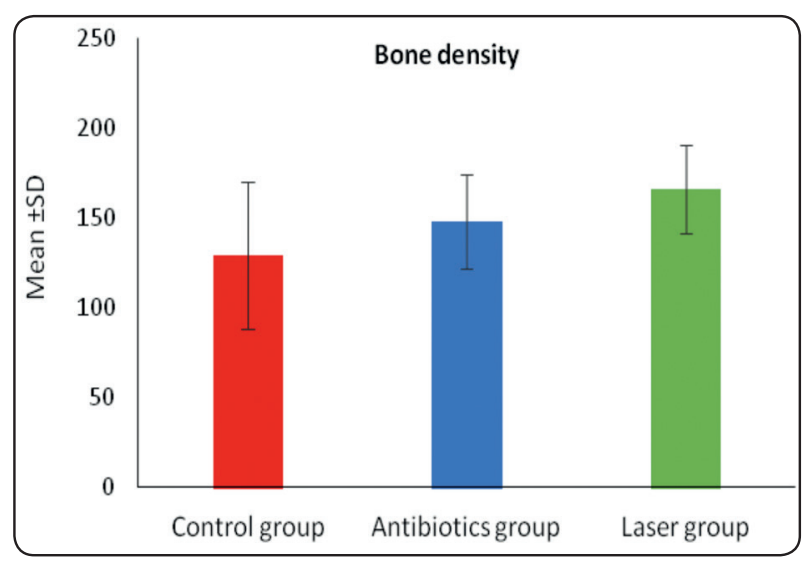

Fig. (6) Graphical representation difference in bone density between three group 


\section{Correlations between bone density and bacterial count results :}

All the collected data of bone density correlated to bacterial count showed no significant differences between groups except in control group there was significant correlation with $\mathrm{p}$ value 0.032 (table 3 and figure 7).

TABLE (3): Correlation between bacterial counts and bone density in the studied groups:

\begin{tabular}{|c|c|c|}
\hline \multicolumn{3}{|c|}{ Correlations } \\
\hline \hline \multirow{2}{*}{$\begin{array}{c}\text { Bacterial count CFU } \\
\text { Infected }\end{array}$} & \multicolumn{2}{|c|}{ Bone density } \\
\cline { 2 - 3 } Control group & -0.851 & $0.032^{*}$ \\
\hline Antibiotics group & 0.168 & 0.750 \\
\hline Laser group & -0.441 & 0.381
\end{tabular}

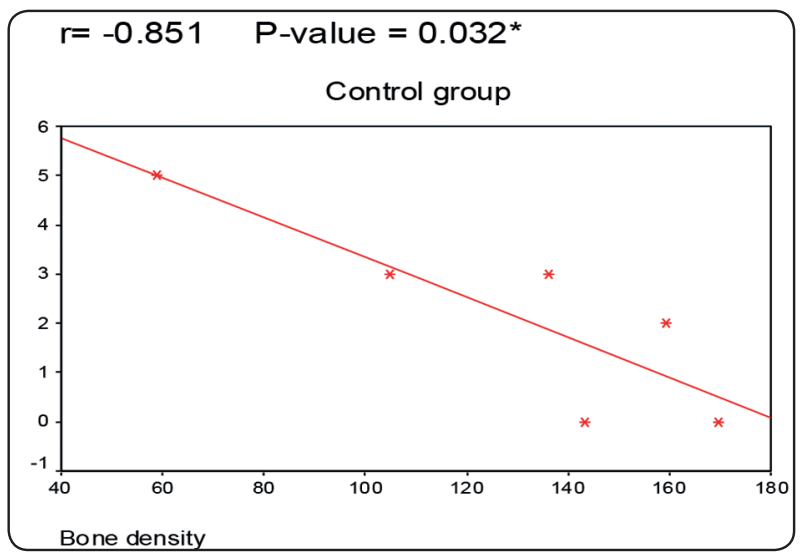

Fig 7: Graphical representation correlation between three group between in bone density and bacterial count .

Correlation between bacterial count and bone density not enough for recommendation.

\section{Recommendation}

We recommended to do more procedure as histopathological examination to confirm between radiographic bone density and bacterial count

\section{ACKNOWLEDGMENTS}

The authors would like to express their gratitude to Dr adel aboelalla and Msc Dr safaa aboelalla for their skilful assistance during the experiment , excellent animal laboratory assistance also for practical guidance at The Veterinary medicine cairo university .

\section{REFERENCES}

1. Lew DP and Waldvogel FA (2004): Osteomyelitis. Lancet; 364: 369-79.

2- Spellberg B and Lipsky BA (2012): Systemic antibiotic therapy for chronic osteomyelitis in adults. Clin Infect Dis 54:393-407

3- Howden BP, Davies JK, Johnson PD, Stinear TP and Grayson ML (2010): Reduced vancomycin susceptibility in Staphylococcus aureus, including vancomycin-intermediate and heterogeneous vancomycin-intermediate strains: resistance mechanisms, laboratory detection, and clinical implications. Clin Microbiol Rev; 23(1): 99-139.

4- Rice D A and Mendez-Vigo L.( 2009): Daptomycin in bone and joint infections: a review of the literature. Arch. Orthop. Trauma Surg. 129:1495-1504.

5- Falagas ME, Giannopoulou KP, Kokolakis GN, and Rafailidis PI (2008) :Fosfomycin: use beyond urinary tract and gastrointestinal infections. Clin. Infect. Dis. 46:1069-1077

6- Joukhadar C, Kugler P S S A, Wennersten C, Moellering RM, and Eliopoulos G.( 2010) :Bactericidal effects of fosfomycin against daptomycin resistant strains of Staphylococcus aureus, poster 16.0” sterreichischer Infektionskongress, Saalfelden, Austria.

7- Grif K, Dierich MP, Pfaller K, Miglioli, PA and Allerberger F.(2001): In vitro activity of fosfomycin in combination with various antistaphylococcal substances. J Antimicrob Chemother; 48, 209-217 .

8- Schintler MV, Traunmüller F, Metzler J, Kreuzwirt G, Spendel S, Mauric O, Popovic M, Scharnagl E, Joukhadar C.(2009):High fosfomycin concentrations in bone and peripheral soft tissue in diabetic patients presenting with bacterial foot infection. J. Antimicrob. Chemother. 64:574-578

9- Popovic M, Steinort D, Pillai S, and Joukhadar C. (2009): Fosfomycin: an old, new friend? Eur. J. Clin. Microbiol. Infect. Dis. 29:127-142. 
10- Poeppl W, Tobudic S, Lingscheid T, Plasenzotti R, Kozakowski N, Georgopoulos A and Burgmann, H. (2011): Efficacy of Fosfomycin in Experimental Osteomyelitis Due to Methicillin-Resistant Staphylococcus aureus. Antimicrobial Agents and Chemotherapy, 55(2), 931-933.

11- Poeppl W, Tobudic S, Lingscheid T, Plasenzotti R, Kozakowski N, Lagler H, Georgopoulos A, Burgmann H. (2011): Daptomycin, fosfomycin, or both for treatment of methicillin-resistant Staphylococcus aureus osteomyelitis in an experimental rat model. Antimicrob Agents Chemother 55:4999-5003.

12- Clinical and Laboratory Standards Institute. (2014). Performance standards for antimicrobial susceptibility testing, 24th informational supplement. Approved standard M100-S24. Clinical and Laboratory Standards Institute, Wayne, PA.

13- Adam Stabholz. (2004): Lasers in endodontics . Dent Clin N Am 48 (2004) 809-832 .

14- Lew DP and Waldvogel FA. (1997): Photodynamic Therapy in the Treatment of Osteomyelitis . J Med, 336, 999-1007.

15- Kaya G, Kaya M, Gürsan N, Kireççi E, Güngörmü M, Balta H.( 2011) The use of 808-nm light therapy to treat experimental chronic osteomyelitis induced in rats by methicillin-resistant Staphylococcus aureus.;24(1):130-4.

16- Duartea , Mendes. P, Marquesb M R , Bezerree JP and Bastosa MF (2009): The Effect of caffeine administration on the early stage of bone healing and bone density . Journal of Archives of oral biology ; 54(8) 717-722.

17- The European Committee on Antimicrobial Susceptibility Testing. (2013):Fosfomycin: rationale for the EUCAST clinical breakpoints, version 1.0.

18- Dagan R, Klugman KP, Craig WA, and Baquero F. (2001). Evidence to support the rationale that bacterial eradication in respiratory tract infection is an important aim of antimicrobial therapy. J. Antimicrob. Chemother. 47:129-140.

19- Gould I M, and MacKenzie FM.( 2002): Antibiotic exposure as a risk factor for emergence of resistance: the influence of concentration. Symp. Ser. Soc. Appl. Microbiol. 31:78S-84S.

20- Huovinen P. (2002): Macrolide-resistant group A streptococcus-now in the United States. N. Engl. J. Med. 346:1243-1245.

21-Poeppl W, Lingscheid T, Bernitzky D, Schwarze UY, Donath O, PerkmannT, Kozakowski N, Plasenzotti R, Reznicek G, Burgmann H. (2014).Efficacy of fosfomycin compared to vancomycin in treatment of implant associated chronic methicillin-resistant Staphylococcus aureus osteomyelitis in rats. Antimicrob Agents Chemother 58:5111-5116.

22-Lingscheid T, Poeppl W, Bernitzky D, Veletzky L, Kussmann M, Plasenzotti R, Burgmann H.( 2015). Daptomycin plus fosfomycin, a synergistic combination in experimental implant-associated osteomyelitis due to methicillinresistant Staphylococcus aureus in rats. Antimicrob Agents Chemother 59:859 -863.

23-Nakazawa H, Kikuchi Y, Honda T, Isago T, and Nozaki M. (2003):Enhancement of antimicrobial effects of various antibiotics against methicillinresistant Staphylococcus aureus (MRSA) by combination with fosfomycin. J. Infect. Chemother. 9:304-309. 